\title{
Efficient Organization of Control Structures in Distributed Implementations
}

\author{
Guido Hogen ${ }^{1}$ and Rita Loogen ${ }^{2}$ \\ 1 RWTH Aachen, Lehrstuhl für Informatik II \\ Ahornstraffe 55, D-52056 Aachen, Germany \\ email: ghogen@zeus.informatik.rwth-aachen.de \\ 2 Philipps-Universität Marburg, Fachgebiet Informatik \\ Hans Meerwein Straße, Lahnberge, D-35032 Marburg, Germany \\ email: loogen@informatik.uni-marburg.de
}

\begin{abstract}
A new technique for the management of control structures in distributed implementations of dynamic process systems is presented. Instead of storing the runtime stacks of parallel processes as linked lists of activation blocks in a heap structure, the local stacks of several parallel processes, which are executed on the same processor element, are stored in an interleaved manner on a single physical stack (within each processor element), called the meshed stack. The technique ensures that there is almost no overhead for the evaluation of single processes due to the parallel environment. In principle, the meshed stack technique is independent of the implemented language. We explain it for the parallel implementation of functional languages.
\end{abstract}

\section{Introduction}

In purely sequential implementations of most programming languages a runtime stack is used for the control of recursive function or procedure calls. Such calls lead to the allocation of control information (activation blocks) on top of the stack. When the call terminates, this information is removed and control is given back to the calling environment. A graph or heap structure is only used for the representation of dynamic data structures.

For parallel implementations, which support dynamic processes, as it is the case in parallel implementations of functional or logic languages, the efficient realization of the runtime stack is one of the most important problems. The use of a global contiguous array of activation blocks is no longer advisable, because each parallel task or process needs its own stack structure. Furthermore a suspension mechanism must be implemented to save the state of tasks which are waiting for the result of some parallel subtask. This led to the idea of allocating runtime structures in a graph or heap instead of on a stack. The runtime stack of each process is implemented as a linked list of activation blocks in the heap [Goldberg, Hudak 86], [Loogen et al. 89], [Augustsson, Johnsson 89], [Kingdon et al. 91]. Spawning of parallel processes yields a tree structure of such linked lists, which represents a so-called cactus stack.

A main advantage of this graph organization is the decentralization which is especially suited for distributed systems, as the bottleneck of a global central 
stack is avoided. Context switches can simply be achieved by redirecting the pointer to the currently active process. Note that heap allocation can be done as efficiently as stack allocation by the incrementation of a heap pointer [Appel 87]. There are however two important disadvantages:

1. the increased organizational overhead for the deallocation (garbage collection) of runtime information and

2. the overhead that is imposed on the purely sequential evaluations within processes.

In this paper we present an alternative management of the runtime structures which tends to support parallel and sequential evaluations equally well. Instead of representing the cactus stack as a linked tree of activation blocks, we allocate the activation blocks, within each processor element of the parallel system, on a single meshed stack, which is organized as a contiguous array, but shared by several processes. The heap is only used for the representation of migrated parallel processes, structured data, closures and suspensions. Evaluation is controlled by the stack. During the sequential evaluation of processes the meshed stack is used as in conventional implementations. Activation blocks are allocated on top of it, when function or procedure calls are executed, and removed, when the calls terminate. Central issues of the new stack technique are the generation and suspension of parallel processes.

The meshed stack technique is independent of the implemented language. We will explain it for the distributed implementation of a functional language.

The paper is organized as follows. In the next section, we introduce a simple first order functional language which is sufficient for the explanation of our approach. Section 3 explains a simple sequential reduction machine which will be the sequential kernel of the parallel implementation. Section 4 introduces parallelism information into the functional language and explains the problems that must be solved to provide a distributed implementation of the parallel functional language. Section 5 is devoted to the explanation of the meshed stack technique. In Section 6 we develop a parallel abstract reduction machine. A special garbage collection method for the meshed stack is described in Section 7. Results from a transputer implementation of our system are contained in Section 8. Finally related work is discussed and some conclusions are drawn.

\section{FUN - A Simple FUNctional Language}

FUN is a first order monomorphically typed functional language with algebraic data structures. We do not consider higher order functions and polymorphism, as the treatment of these features is orthogonal to our implementation technique.

A $F U N$-program consists of a finite set of defining rules for function symbols. $F S$ denotes the set of function symbols defined in a program. The defining rule for an element $f \in F S$ has the form: $f\left(x_{1}, \ldots, x_{n}\right):=e$, where $x_{i}$ are variables and $e$ is a FUN-expression. The definition of FUN-expressions is given in Fig. 1.

Function symbols in applications may be basic operation symbols $\phi \in \Omega$, data constructors $\phi \in \Gamma$ or defined function symbols $\phi \in F S$. case-expressions define a complete case analysis on the top level constructor of a data structure expression. The predefined projection operator enables the selection of data 


\begin{tabular}{|c|c|c|}
\hline 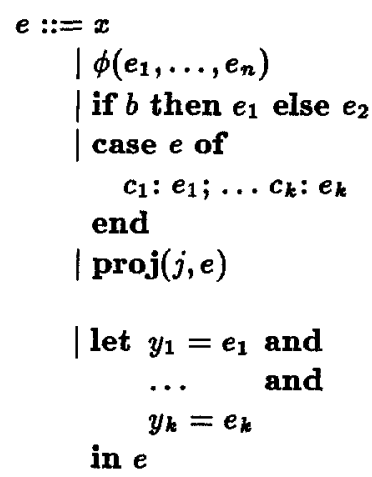 & $\begin{array}{l}\% x \in \operatorname{Var}^{t} \\
\% \phi \in(\Omega \cup \Gamma \cup F S)^{t_{1} \times \ldots \times t} \\
\% b \in E x p^{b o o l}, e_{1}, e_{2} \in E_{x p}{ }^{t} \\
\% e \in E x p^{d}, \\
\% c_{i} \in \Gamma^{\cdots \rightarrow d}, e_{i} \in E x p^{t} \\
\% e \in E_{x p}^{d} \\
\% y_{i} \in \operatorname{Var}^{t_{i}}, e_{i} \in E x p^{t_{i}} \\
\% e \in \operatorname{Exp}^{t}\end{array}$ & $\begin{array}{l}{ }^{n \rightarrow t}, e_{i} \in E x p^{t_{i}} \\
\text { conditional expr. } \\
\text { case expression } \\
\text { projection to } j \text { th } \\
\text { component } \\
\text { local definitions }\end{array}$ \\
\hline
\end{tabular}

Fig. 1. FUN-Expressions

structure components. let-expressions define sharing of arbitrary subexpressions. We always assume that rules and expressions are well-typed.

The semantics of FUN programs are the usual lazy reduction semantics with strict interpretations of basic operations. The program rules are used as rewrite rules for the evaluation of applications of defined function symbols.

In our example programs we assume the predefined base types integer and boolean with the usual arithmetic and boolean operation symbols, for which we allow infix notation.

The definition of the function symbol tower assumes the data structure tree with constructors leaf of type int $\rightarrow$ tree and node of type tree $\times$ int $\times$ tree $\rightarrow$ tree. append is the well known list concatenation function.

$$
\begin{array}{r}
\operatorname{psum}(l, h):=\begin{array}{l}
\text { if } l=h \text { then } l \text { else if } l+1=h \text { then } l+h \\
\text { else let } m=(l+h) / 2 \text { in psum }(l, m)+p \operatorname{sum}(m+1, h)
\end{array} \\
\begin{array}{r}
\text { tower }(n, \text { sour, help, dest }):= \\
\text { let move }=10 * \text { sour }+ \text { dest } \\
\text { in if } n=1 \text { then leaf }(\text { move }) \\
\text { else node }(\text { tower }(n-1, \text { sour, dest, help }), \text { move, } \\
\quad \operatorname{tower}(n-1, \text { help, sour, dest }))
\end{array} \\
\begin{array}{r}
\text { append }\left(l_{1}, l_{2}\right):= \\
\text { case } l_{1} \text { of nil }: \quad l_{2} ; \\
\text { cons }: \operatorname{cons}\left(\operatorname{proj}\left(1, l_{1}\right), \text { append }\left(\operatorname{proj}\left(2, l_{1}\right), l_{2}\right)\right)
\end{array}
\end{array}
$$

\section{Implementation of Functional Languages}

In this section we shortly describe the structure and the behaviour of a simple reduction machine for the implementation of functional languages. This reduction machine is the sequential kernel of the parallel implementation.

\subsection{Structure of the Reduction Machine}

The store of the reduction machine is illustrated in Fig. 2. Its central component is the function stack which contains the activation blocks of recursive function calls. Structured data, basic values, and arguments, whose evaluation 


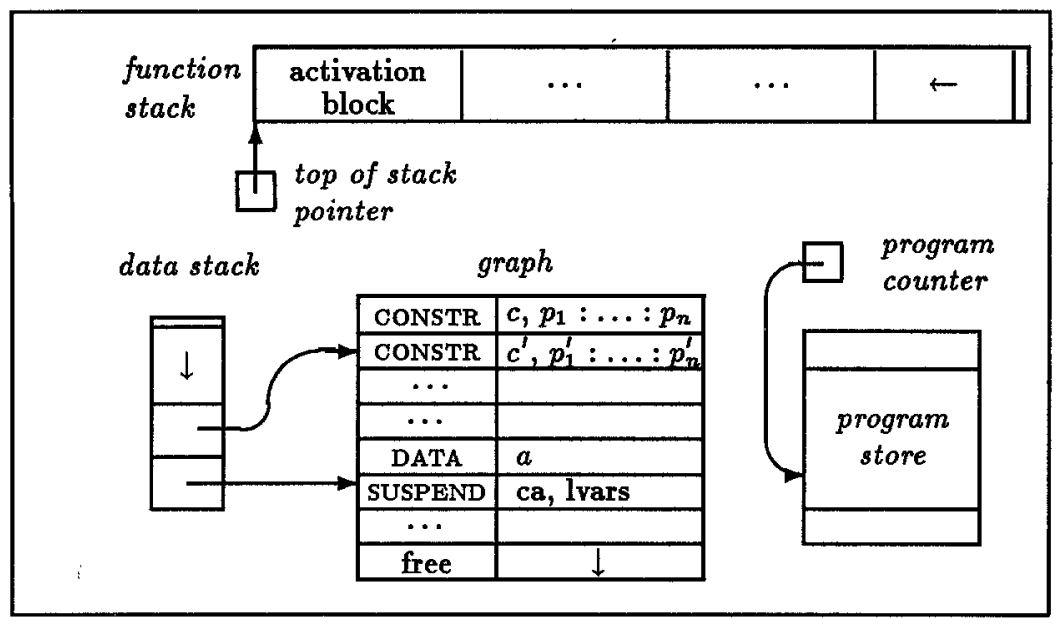

Fig. 2. Structure of a Sequential Reduction Machine

is postponed in case of a lazy evaluation strategy, are represented in the graph. Consequently, we distinguish between constructor nodes, data nodes, and suspension nodes (see Fig. 2). Constructor nodes contain the constructor name $c$ and pointers to the graph representation of the components. Data nodes simply contain a basic value. Suspension nodes contain a code address $c a$, which is the address, at which the code for the evaluation of the delayed or suspended argument starts, and a list lvars of parameters to which some instructions in the code may refer.

\subsection{Behaviour of the Reduction Machine}

In order to evaluate a function application, the addresses of the graph representations of the arguments are written on top of the data stack and a function call is executed. The instruction CALL $(c a, n, k)$ creates a new activation block on top of the function stack, moves $n$ pointers to the function arguments from the data stack to the function stack, reserves $k$ cells for local variables, writes the value of the instruction pointer as return address and the dynamic link to the previously active activation block into the new activation block and finally jumps to the program address ca.

The code for each function $f$ consists of the code for the body expression $e$ of $f$ 's defining rule and a RETURN instruction:

\section{Code for $f$ : exptrans $(e)$; RETURN.}

The return instruction terminates a function call by removing the top level activation block from the stack and jumping to the saved return address. The result of the function call is given on top of the data stack.

The compilation of expressions is shown in Fig. 3. For simplicity, we do not consider any optimization techniques as e.g. tail recursive function calls.

The reduction machine is capable of carrying out an innermost and a lazy strategy. Which strategy is chosen to execute a program, depends on the compilation of argument expressions. In case of an innermost strategy, arguments are translated by the exptrans-scheme. This implies that expressions are evaluated 


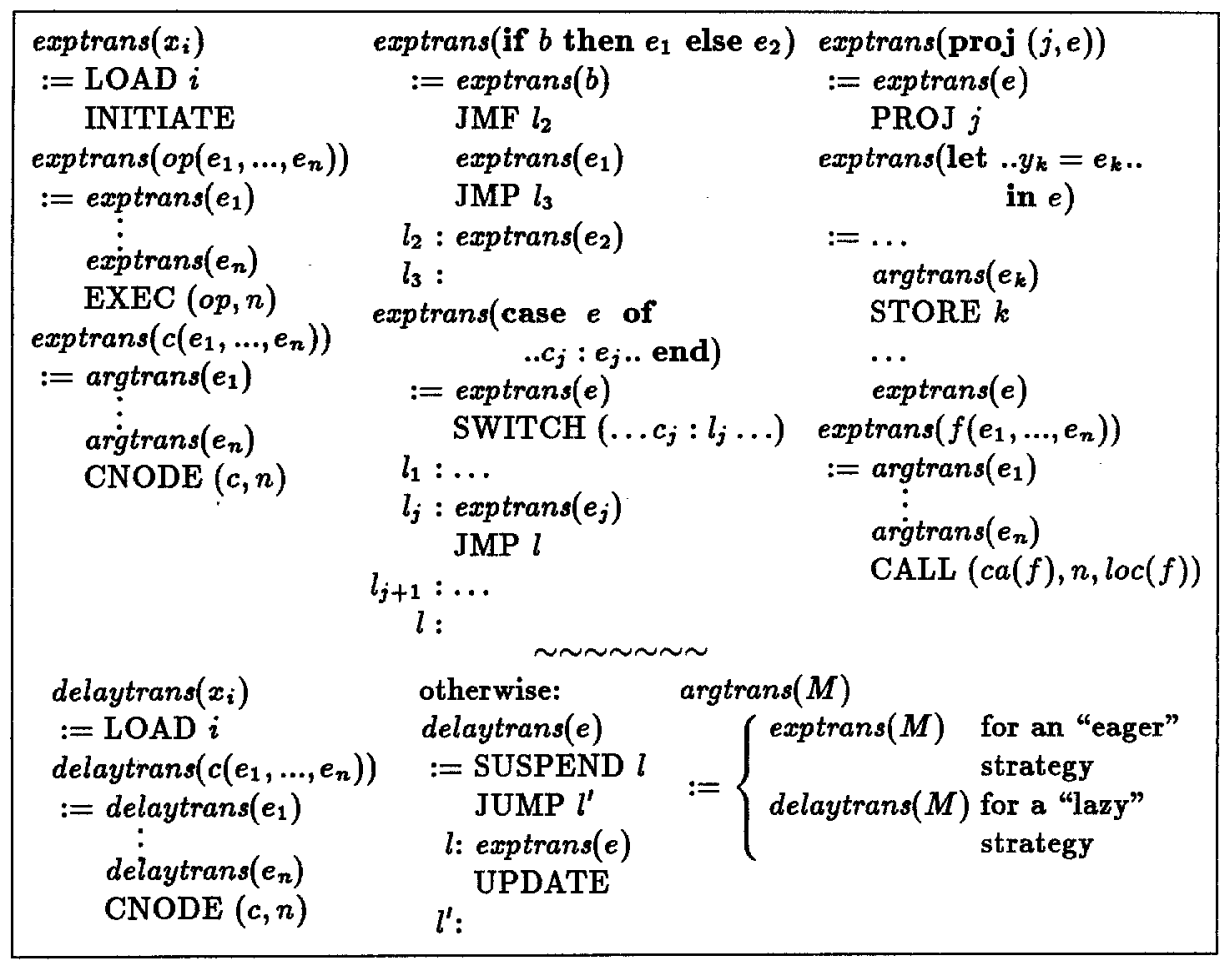

Fig. 3. Sequential Code for Expressions

to normal form and INITIATE instructions, which are necessary to force the evaluation of suspended arguments, can be omitted. In case of a lazy evaluation strategy, arguments are translated by the delaytrans-scheme, which generates suspension nodes for the representation of arguments not evaluated yet, using the instruction SUSPEND. Furthermore, code for the evaluation of the suspension is generated using the exptrans-scheme. This code is concluded by the instruction UPDATE, which writes the result of the evaluation into the suspension node and gives control back to the calling environment.

\section{Parallelism in Functional Languages}

The implicit parallelism within FUN-programs consists of the reduction of independent subexpressions which is possible due to the side effect free nature of the reduction semantics. The eager evaluation strategy allows the parallel evaluation of arbitrary subexpressions. In case of the lazy reduction strategy, strictness analysis can be used to find out subexpressions which can be safely evaluated in parallel. Usually annotations or special syntactic constructs are used to show the implicit parallelism within programs [Goldberg, Hudak 86], [Augustsson, Johnson 89]. An automatic parallelizing compiler for lazy functional languages is described in [Hogen et al. 92]. This compiler translates functional programs into a parallelized form where the construct

letpar $y_{1}=f_{1}\left(e_{11}, \ldots, e_{1 n_{1}}\right)$ and $\cdots$ and $y_{p}=f_{p}\left(e_{p 1}, \ldots, e_{p n_{p}}\right)$ in $e$ 
is used to indicate parallelism. Such a letpar-expression means that the function applications $f_{i}\left(e_{i 1}, \ldots, e_{i n_{i}}\right)$ may be evaluated in parallel with the main stream of evaluation, $e$. Whether a parallel evaluation really takes place at runtime, depends on the workload of the distributed system. Evaluation of a letparexpression first leads to the parallel activation of the function applications. Then the evaluation of the body $e$ proceeds until the result of a parallel subexpression, represented by $y_{j}$, is needed but not available yet. In this case, the evaluation of $e$ will be suspended until the parallel subprocess sends its result. In the meantime, the processor should be free to evaluate some other process.

The resulting process system is hierarchical. The execution of a parallelized functional program starts with the evaluation of a main expression. letparexpressions generate parallel subprocesses, that can be executed on other processor elements. By the execution of parallel processes, further processes may be generated. When a process terminates, its result is communicated to the father process, i.e. the process that generated the subprocess, and all other processes waiting for the result.

A parallelization of the first two example functions of Section 2 is:

$$
\begin{aligned}
& \operatorname{psum}(l, h):=\text { if } l=h \text { then } l \text { else if } l+1=h \text { then } l+h \\
& \text { else let } m=(l+h) / 2 \text { in letpar } y_{1}=p \operatorname{sum}(l, m) \\
& \text { in } \operatorname{psum}(m+1, h)+y_{1} \\
& \text { tower(n, sour, help, dest }):= \\
& \text { let move }=10 * \text { sour }+ \text { dest } \\
& \text { in if } n=1 \text { then leaf (move) } \\
& \text { else letpar } y_{1}=\text { tower }(n-1, \text { help, sour, dest }) \\
& \text { in node (tower } \left.(n-1 \text {, sour, dest, help }) \text {, move, } y_{1}\right)
\end{aligned}
$$

The process system that will be created for the expression psum $(1,6)$ is shown in Fig. 4. It shows already the shape of the cactus stack the parallel system has to cope with. Most of the existing parallel implementations of functional languages generate for each recursive function call a new activation block, called task node or VAP (variable sized application) node, in the heap [Augustsson, Johnson 89], [Loogen et al. 89], [Kingdon et al 91]. The activation blocks in the heap contain all the information and substructures that are necessary for the evaluation of the corresponding function call. A parallel evaluation can simply be achieved by the migration of a block to another processor element.

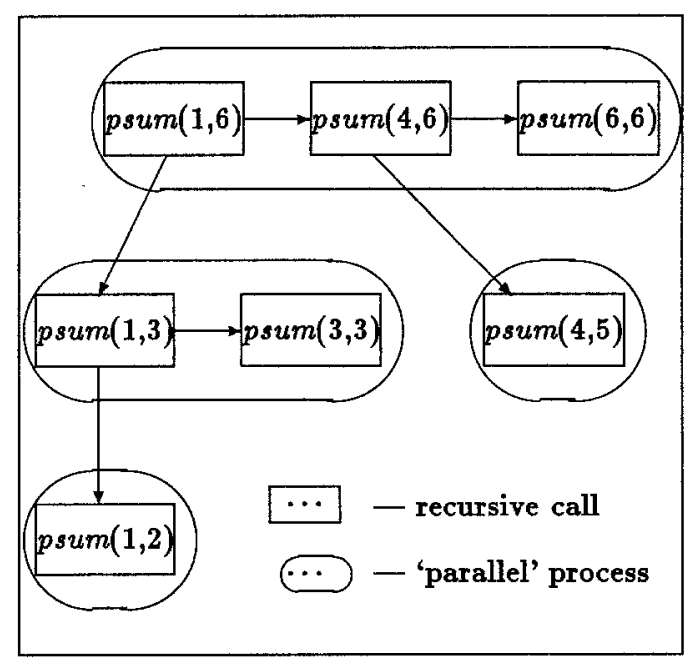

Fig. 4. Recursive Calls and Parallel Processes of the psum-Program 
The dynamic extension of activation blocks in case of tail recursive function calls can be avoided by the use of the stacklessness analysis of [Lester 89a], which allows to create activation blocks large enough to evaluate all tail recursive calls that may occur in a reduction sequence.

In this paper we claim that this "linked list"-realization of the cactus stack is not natural, because it does not support the sequential evaluations within processes in an optimal way. The knowledge about the stack behaviour of the various local stacks is lost and general garbage collection techniques must be used to collect the activation blocks of terminated tasks.

[Goldberg, Hudak 86] already pointed out that the graph allocation of activation blocks should be avoided for purely sequential functions which do not spawn parallel subtasks and may not be suspended due to parallel subtasks. They proposed a hybrid system which assumes an analysis and decomposition of the program into sequential and non-sequential functions. For sequential functions an ordinary stack-based evaluation is arranged. For non-sequential function calls the more expensive allocation of runtime information in the heap must be chosen to support parallel evaluations.

In the following, we will present a new stack technique that is a combination of the heap organization of parallel processes and the stack management of sequential evaluation sequences. It works for arbitrary dynamic process systems.

\section{The Meshed Stack Technique}

From an abstract point of view, each parallel process will be executed on a sequential reduction machine, which is capable of the generation of new parallel processes. Thus, each process works on its own function stack area. For sequential recursive function calls, new activation blocks are generated on top of this private function stack. Parallel processes are spawned by generating a special node in the graph, which serves as a placeholder for the result of the process. The characteristic information of the process is embedded into a so-called process message, which contains also the address of the placeholder node. This message will be sent to another reduction machine. The placeholder for the result of the parallel process can be seen as a graph representation of the migrated evaluation.

In a concrete parallel system there will, of course, be only a finite number of processor elements. As processes are created dynamically, i.e. there is no restriction on the number of processes, this implies that each reduction machine within a parallel environment must be capable of multitasking. It must be possible to evaluate several parallel processes in a single reduction unit. In particular, a machine must be able to organize the interleaved evaluation of several active processes.

The main problem is how to organize the runtime information of several parallel processes in a space- and time-efficient way. One possibility is the pure graph organization which leads to a linked-list realization of local function stacks, which we already rejected. Another possibility is the allocation of private contiguous stack areas within a reserved memory area for the function stack. This approach is very time-efficient, but it leads to a fragmentation of the function 
stack area and there will be a restriction on the number of parallel processes which can be executed in a single reduction unit or there must be some mechanism to split stack areas if more processes than available stack areas are created. Furthermore there must be some mechanism for the dynamic extension of stack areas, if processes run out of stack space and there is still free space.

Because of these difficulties, we decided to try another approach which originated from the organization of runtime information in the Warren Abstract Machine (WAM), which has been developed for the implementation of logic languages [Warren 83]. This might be surprising, but there is the following analogy. In the WAM the runtime information of several alternative computation sequences is kept on a single stack. This stack is not a stack in the conventional sense. Due to the information that is kept for alternative computation branches, the active activation block (environment) is not always on top of the stack and on termination of "procedure" calls the activation blocks can only be removed from the stack if they are on top of it.

Our meshed stack keeps the runtime information of several parallel processes on a single stack. Its main property is that the current activation block need not be on top of the stack. New activation blocks will always be generated on top of the stack, but if a function call terminates and its activation block is not on top of the stack, it will not be removed, but simply marked as garbage. If a parallel process is suspended, because it has to wait for some information that is not locally available, its activation blocks are kept on the stack until its evaluation can continue. In the meantime, the reduction machine can proceed with the evaluation of another parallel process, whose activation blocks will simply be allocated on top of the activation blocks of the suspended process. If the new process will eventually be suspended and the process is reactivated, newly created activation blocks of this evaluation will be allocated on top of the activation blocks of the previous evaluation. Thus we will get a mixture of activation blocks belonging to different parallel processes on the stack, which is the reason for calling it a meshed stack. This explains the main idea of the meshed stack technique.

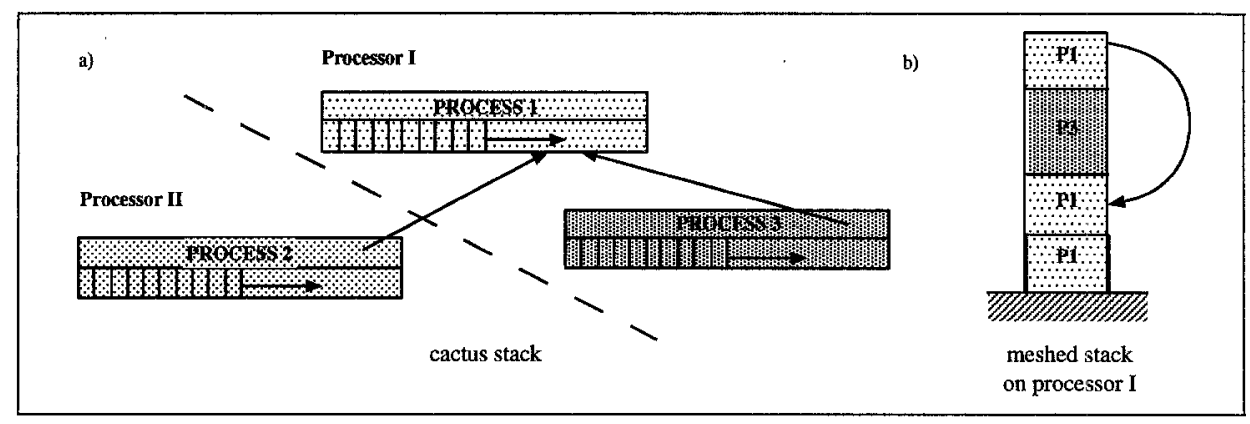

Fig. 5. Implementation of a Cactus Stack on a Meshed Stack

In Fig. 5 a cactus stack and its implementation with a meshed stack is shown. In the cactus stack each node represents one parallel task including sequential function calls. So each node represents a number of activation blocks. Process 
1 and 3 are executed in the same reduction machine. If we look at the meshed stack of this reduction machine, we find the activation blocks of these processes interleaved.

But, what happens when function calls terminate and the corresponding blocks can be deallocated. The deallocation of blocks on top of the stack is of course no problem. Inner activation blocks which can be removed are marked as garbage. They are collected, i.e. removed, as soon as there is no valid, i.e. active or suspended activation block on top of them. If the stack runs out of space, a special garbage collector is invoked. This garbage collector simply crunches the stack in place in one pass. Details will be given in Section 7.

\section{Development of a Parallel Reduction Machine}

In this section we develop a parallel reduction machine by embedding extended sequential reduction machines in a parallel environment.

\subsection{General structure}

The parallel reduction machine consists of a finite number of identical processor elements, each with a local storage. An interconnection network enables the processor elements to communicate by exchanging messages. Each processor element consists of two independent processing units, which communicate via the exchange of messages in a local shared memory (see Fig. 6).

- The communication unit handles the organization of the parallel aspects of the reduction process.

- The reduction unit is a sequential reduction machine which has been extended by capabilities for multitasking and for the processing of messages.

The main task of the communication unit is the distribution of parallel processes and the organization of the message traffic. We will not go into the details of the communication processor, as task distribution and routing strategies are out of the scope of this paper. We assume a simple static routing scheme and a passive process distribution strategy, i.e. processor elements without work ask their neighbouring processor elements for processes. In its local store the communication processor maintains a process queue which contains parallel processes which have been generated by the reduction unit and a static table for the routing of messages from and to other processor elements.

\subsection{Process Management in the Reduction Units}

The store of the sequential reduction machine is extended by a ready queue, which holds the local suspended processes which are ready to be re-activated (see Fig. 6). The function stack is now organized as a meshed stack.

The data stack will be divided into local data stack areas for each activation block. This is necessary to avoid the saving of data stack entries on process switches. Each function call gets an activation block on the function stack and its own "private" data stack. For allocation purposes one has to know the maximum storage demands of each local data stack. These can be estimated during compile 


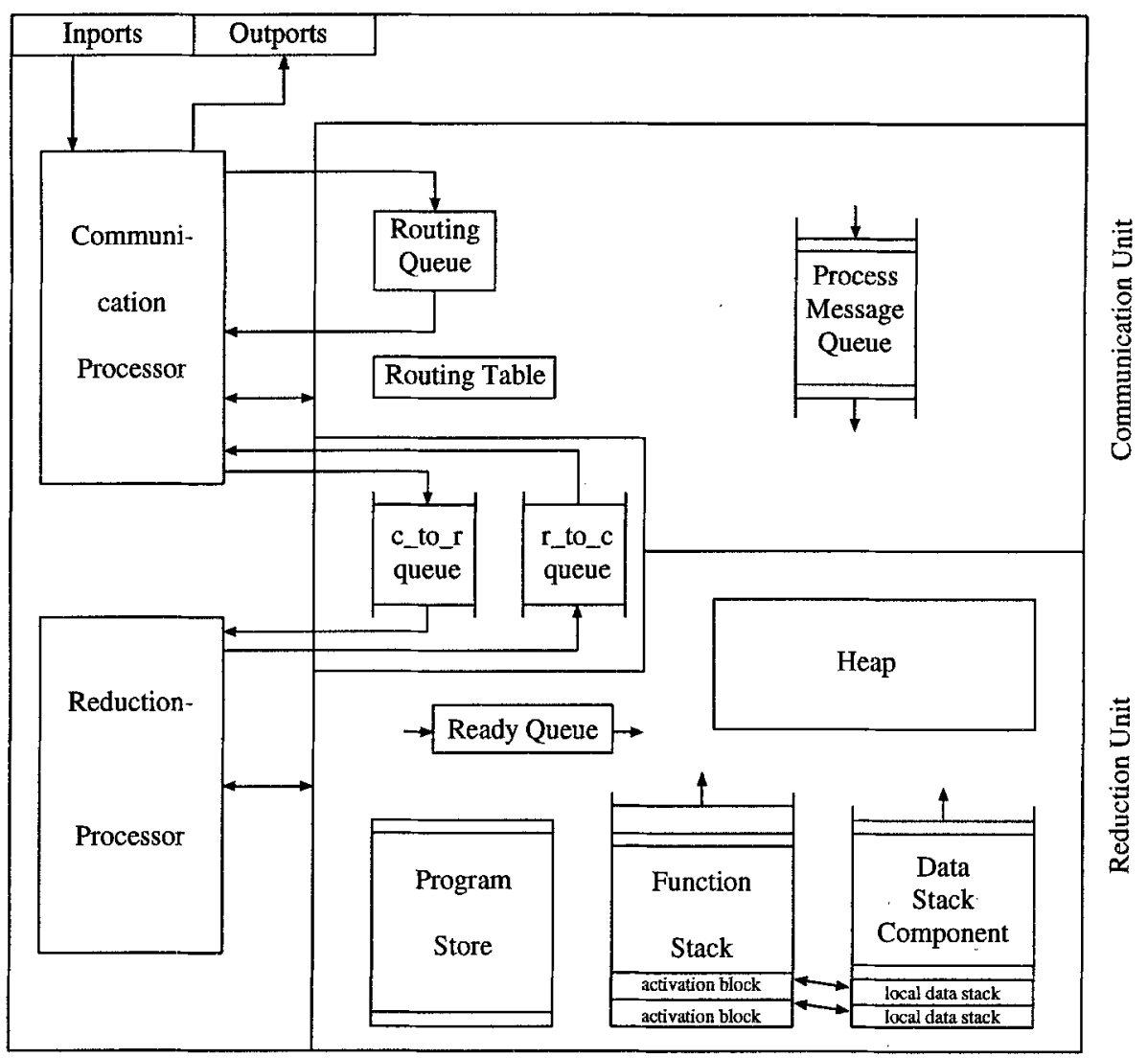

Fig. 6. Global Structure of a Single Processor Unit

time and passed as a parameter in the instructions which generate new activation blocks, as e.g. CALL. The heap component is extended by placeholder nodes for the results of parallel processes.

\section{Process creation}

For spawning a parallel function call a new instruction, similar to the CALLinstruction, is introduced:

TASK $(c a, n, k$, size) produces a process that can be executed in parallel. $c a$ is the code address, $n$ the number of arguments, $k$ the number of local variables in the definition of the corresponding function and size the maximal data stack size that will be necessary during the execution of the function. In the heap component, a placeholder node for the result is created. A process message containing the code address and parameters of the parallel function call and the address of the placeholder node is sent to the communication unit. The computation of the actual task proceeds, until there is a demand for the result of a parallel subtask.

The communication unit stores the process message in its process queue, as processes are only distributed to other processor elements on demand. If the 
reduction unit runs idle, the communication unit can satisfy its workrequest by an entry from its own process queue before asking other processor elements. In this case, a parallel process is executed on its creation processor.

Let us consider now program compilation: When reaching a letpar-construct, parallel tasks for all function calls on the right hand side are created by the TASK-instruction. The addresses of the placeholder nodes are stored into the corresponding local variable positions within the activation block.

$$
\begin{aligned}
& \text { exptrans } \llbracket \text { letpar } y_{m+1}=f_{1}\left(e_{11}, \ldots, e_{1 l_{1}}\right):=\operatorname{pcode}\left(f_{1}\left(e_{11}, \ldots, e_{1 l_{1}}\right)\right) \\
& \text { and } \ldots . \quad \text { STORE } m+1 \text {; } \\
& \text { and } y_{m+p}=f_{p}\left(e_{p 1}, \ldots, e_{p l_{p}}\right) \\
& \text { in } e \rrbracket \\
& \text { pcode }\left(f_{p}\left(e_{p 1}, \ldots, e_{p l_{p}}\right)\right) \\
& \text { STORE } m+p \text {; }
\end{aligned}
$$

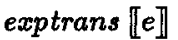

$$
\begin{aligned}
& \text { where } \operatorname{pcode}\left(f\left(e_{1}, \ldots, e_{j}\right)\right):=\left\{\begin{array}{l}
\operatorname{argtrans} \llbracket e_{1} \rrbracket \\
\vdots \\
\operatorname{argtrans} \llbracket e_{j} \rrbracket \\
\operatorname{TASK}(c a(f), j, \operatorname{loc}(f), \operatorname{dsize}(f))
\end{array}\right.
\end{aligned}
$$

\section{Process suspension}

The suspension of processes has to enable a context switch to another process.

When a process encounters a situation where a value is needed but not available yet, i.e. when a process accesses a placeholder node, it has to wait until the placeholder node is overwritten by the result. In the meantime the reduction unit should proceed with another process, which can be taken from the ready queue of reactivated processes or from the process queue in the communication unit. The following instruction performs the suspension of a process.

WAIT checks if the top entry of the data stack is evaluated. If not, the active process is suspended until the result is available. Therefore, the stack address of the current activation block is added to the list of waiting tasks within the placeholder node of the argument. When the result is delivered, the waiting list of a placeholder node is moved to the ready queue.

The WAIT-instruction is used for the compilation of variables in a context where the result of the variable is needed.

$$
\text { exptrans } \llbracket y_{i} \rrbracket:=\left\{\begin{array}{l}
\text { LOAD } i ; \\
\text { WAIT; } \\
\text { LOAD } i ; \\
\text { INITIATE; }
\end{array} \text { if } y_{i}\right. \text { is a variable introduced using letpar }
$$

We have to distinguish between variables introduced by the letpar-construct and others (formal parameters and local variables introduced by let). The latter are always locally available and can be accessed directly. The result of parallel processes may not be available, so one has to suspend the actual evaluation.

\section{Process termination}

As in the sequential machine, the RETURN instruction terminates a function call. It returns the result to the calling process and marks the actual activation 
block and data stack as garbage. Due to parallel execution, the calling task can be local (so one can put the pointer onto the calling data stack and continue computing the calling task) or global (the result is sent to the placeholder node on the other processor element).

\subsection{Message Handling in the Reduction Units}

During the execution of processes, the reduction units will produce

- process messages when parallel processes are spawned,

- memory request messages when graph nodes which are allocated in the heap of another reduction unit are needed,

- answer messages in order to reply to process and memory request messages.

- work requests to ask the communication unit for new processes.

In detail, the reduction units behave as follows on incoming messages:

A process message represents a parallel ready-to-run process. It will be received after a work request message has been sent to the communication processor. A new activation block is created and its stack pointer is added to the ready queue. One cannot start the process directly (although demanded by the work request) because in the time between the work request and the task message, a result could have been delivered which triggered other activations. Also, due to different scheduling strategies, several processes could be delivered on demand.

A memory request message forces the reduction unit to look up a value for another processor element and to send the value in form of an answer message back to the inquiring processor. If the requested graph node is a suspension, the reduction unit will first initiate its local evaluation. The request message will be answered as soon as this evaluation finishes.

Finally, answer messages overwrite the placeholder nodes and activate the waiting tasks.

\section{Garbage-Collection in the Meshed Stack}

Due to the distinction between control information (in the stack component) and data information (stored in the heap component), one can handle each component with an appropriate garbage collection mechanism. E.g., the problem of data sharing needs only to be handled by the garbage collector of the heap component and causes no unnecessary administration overhead in the stack garbage collector. One major advantage of our approach is the fact, that the knowledge of the special stack structure can be used to develop a simple and efficient garbage collector for this component.

In general, activation blocks can only be released immediately from the topof-stack pointer until one finds a non-garbage block. Inbetween the stack, there may be still some "holes" left (see Fig. 7). If we had an infinite stack space, the above strategy would be sufficient to eventually release all blocks. Since our stack space is limited, the stack can run out of space while there are still garbage holes within the stack.

Each block contains an individual block id, all references to the activation block are made throngh this id. The actual address of the block can be 
found in a table which maps id's to stack addresses. If the garbage collector is invoked, the stack is simply crunched in place, deleting the holes. The main procedure is outlined in Fig. 8. Starting from the bottom, garbage blocks are skipped and their id's are released. Once a valid block is encountered, it is moved "down" the stack and the address entry in the table is updated ${ }^{1}$. This process is iterated until the top of the stack is reached.

References to the stack occur only locally as waiting task id's, attached to the placeholder nodes. So, at the moment a waiting task is activated, it looks up its actual stack position in the table. Therefore the garbage collection process is completely transparent to the rest of the environment. The indirection introduced by the address table causes no significant overhead, since the mapping of id's to actual addresses is only resolved during context switches.

Details of the stack garbage collector are given in [Schmidt 93].

Fig. 8. The Main Procedure for Garbage Collecting the Stack

\section{Implementation Results}

The implementation of an interpreter version of the machine on a transputer system under Helios $\mathrm{C}$ is still in progress. Therefore, we will only present runtime results for some simple example programs.

In a real system the length of the process message queue, which is managed by the communication unit is limited and may overflow. First of all, the question

${ }^{1}$ The functions size, id and garbage yield the size, id and garbage information of the actual block, resp. nextblock sets the pointer to the activation block on top of the actual one. 
arises, what to do in that case. Obviously, too much tasks are generated. So we introduce a flag $p m q_{-}$full which is set by the communication unit and can be read by the reduction unit. If the reduction process encounters a TASK-instruction, it first checks the boolean flag. If the queue is full, instead of sparking a new task, a CALL-instruction is simulated, causing the generation of a local activation block. The introduction of this flag has two interesting side-effects:

1. If only one processor is involved (the total amount of processors in the system is known by every processor), the pmqfull-flag can simply be set to true, thus suppressing any task generation.

2. The maximum number of process message queue entries can be changed during runtime according to statistics about the system behaviour. So the system can adjust itself dynamically and the generation of too much tasks flooding the system can be prevented.

In our system we have implemented the first feature, which results in a runtime behaviour of a parallel program on one processor that is very similar to the sequential version of the program.

In Fig. 9 runtime measurements (in seconds) of the parallel example programs of Section 4 and the well known $n f i b$-program

$n f i b(x):=$ if $x<2$ then 1 else letpar $y_{1}=n f i b(x-2)$ in $n f i b(x-1)+y_{1}+1$ on 1, 2 and 4 processor elements $(P E)$ are presented.

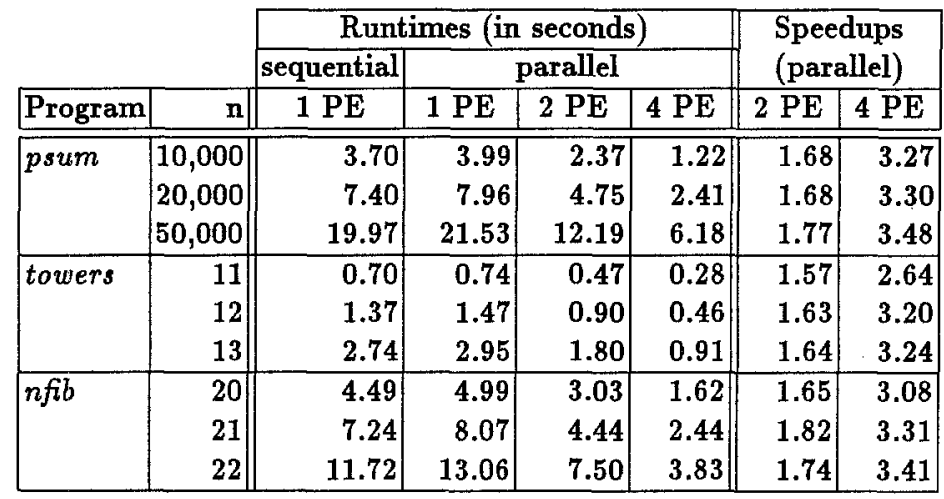

Fig. 9. Runtimes and Speedups for Example Programs on the Parallel Machine

\section{Related Work and Conclusions}

Most parallel implementations of functional languages rely on the graph-based model of reduction $(\langle\nu, G\rangle$-machine of [Augustsson, Johnsson 89], the HDGmachine of [Kingdon et al. 91], ALFALFA. [Goldberg, Hudak 86], PAM [Loogen et al. 89]). There have been attempts to incorporate stack techniques ([Koopman et al. 90], [Peyton-Jones 91]), but only on the sequential level. We presented an alternative extended stack technique for the management of control structures in distributed implementation of dynamic process systems. The technique has been presented in the context of functional languages, but in principle it is independent of the chosen programming language. 
A similar approach has been taken in [Hofman et al. 92], but there only activation blocks on top of the stack are allowed to be executed. So inner activation blocks may be ready to be reactivated, but are blocked by the top-of-stack task. Note that they consider a fork/join task model. In our environment, where sharing of subtasks is allowed, this approach could result in deadlock situations. Nevertheless, their approach yields a good cache behaviour, which should also be observed in our machine.

Our technique allows for a special efficient garbage collection of control structures. For garbage collection of the heap, the approach of [Lester 89b], a combined scheme of a (local) copying and a (global) reference counting garbage collector, seems appropriate and will be implemented in the future.

\section{References}

[Appel 87] A.W. Appel: Garbage Collection can be faster than stack allocation, Information Processing Letters, 21(4):275-279, 1987.

[Augustsson, Johnsson 89] L. Augustsson, Th. Johnsson: Parallel Graph Reduction with the $\langle\nu, G\rangle$-Machine, Conf. on Functional Progr. Lang. and Comp. Arch. (FPCA), Addison-Wesley 1989.

[Goldberg, Hudak 86] B. Goldberg, P. Hudak: ALFALFA - Distributed Graph Reduction on a Hypercube Multiprocessor, Workshop on Graph Reduction, LNCS 279, Springer 1986.

[Hogen et al. 92] G. Hogen, A. Kindler, R. Loogen: Automatic Parallelization of Lazy Functional Programs, Europ. Symp. on Progr. (ESOP '92), LNCS 582, Springer 1992.

[Hofman et al. 92] R. Hofmann, K. Langendoen, W.G. Vree: Scheduling Consequences of Keeping Parents at Home, Proceedings of ICPADS'92, December 1992.

[Kingdon et al. 91] H. Kingdon, D. Lester, G. Burn: The HDG-Machine: a highly distributed graph-reducer for a transputer network, Computer Journal 34 (4), 1991.

[Koopman et al. 90] P. Koopman, M. van Eekelen, E. Nöcker, J. Smeetsers, R. Plasmeijer: The ABC-machine: A Sequential Stack-based Machine for Graph Rewriting, Technical Report 90-22, University of Nijmegen 1990.

[Lester 89a] D. Lester: Stacklessness: Compiling Recursion for a Distributed Architecture, Conf. on Functional Progr. Lang. and Comp. Arch. (FPCA), Addison-Wesley 1989.

[Lester 89b] D. Lester: An Efficient Distributed Garbage Collection Algorithm, Conf. on Parallel Arch. and Lang. Europe (PARLE) 1989, LNCS 365, Springer 1989.

[Loogen et al. 89] R. Loogen, H. Kuchen, K. Indermark, W. Damm: Distributed Implementation of Programmed Graph Reduction, Conf. on Parallel Arch. and Lang. Europe (PARLE) 1989, LNCS 365, Springer 1989.

[Peyton-Jones 91] S.L. Peyton-Jones: The Spineless tagless G-machine: Second Attempt, Proceedings of the Workshop on Parallel Impl. of Functional Lang. , Technical Report CSTR 91-07, University of Southhampton 1991.

[Schmidt 93] A. Schmidt: Garbage Collection of Meshed Stacks, Diploma Thesis, RWTH Aachen 1994.

[Warren 83] D.H.D. Warren: An abstract Prolog instruction set, Technical Report 309, SRI International 1983. 